\title{
Role of Diffusion Weighted Imaging in Characterization of Intracranial Rim Enhancing Lesions
}

\author{
Thapa A, Suwal S, Chataut D, Subedi K \\ Department of Radiology and Imaging, TU Teaching Hospital, Maharajgunj, Kathmandu, \\ Nepal
}

Received: December 5, 2018

Accepted: February 30, 2019

Published: December 31, 2019

Cite this paper:

Thapa A, Suwal S, Chataut D, Subedi K. Role of Diffusion Weighted Imaging in Characterization of Intracranial Rim Enhancing Lesions. Nepalese Journal of Radiology 2019;9(14):17-23.http://dx.doi.org/10.3126/njr. v9i2.27417

\section{ABSTRACT}

Introduction: The differentiation of rim enhancing abscess from high grade necrotic lesions is difficult in conventional imaging. Our purpose was to assess the role of diffusion weighted imaging of intracranial rim enhancing lesions to differentiate the etiologies.

Methods: Fifty one patients, 32 male and 19 female with mean age of 48.47 years and with rim enhancing intracranial lesions in magnetic resonance imaging, who underwent surgery from January 2012 to December 2012, were studied for different characteristics of the lesions in DWI and pathologically correlated the observed findings.

Results: Out of 58 rim enhancing lesions 21 were primary brain tumor, 18 abscess, 14 metastasis and 5 neurocysticercosis. Twenty lesions had restricted diffusion in center, 22 lesions had thin smooth enhancing rim and 23 lesions were with low T2 complete rim. Diffusion restriction at non enhancing center of the lesion and thin smooth enhancing rim have sensitivity and specificity of $88.89 \%$ and $90.24 \%$ ( $p<0.0001$ ) for brain abscess and $83.33 \%$ and $80.49 \%$ (p $<0.0001)$ for other lesions. ADC ratio of center to that of normal white matter showed sensitivity and specificity of $88.9 \%$ and $90 \%$ respectively $(p<0.0001)$ at cut off point of 1.09 . Lesions with thin smooth rim enhancement with diffusion restriction in non- enhancing center are $100 \%$ specific for brain abscess.

Conclusions: On studying the different MRI characteristics of rim enhancing lesions, combining enhancement characteristic with DWI is more helpful in coming to the proper diagnosis.

Keywords: Brain Abscess; Neurocysticercosis; White Matter

\section{INTRODUCTION}

Correspondence to: Dr. Sundar Suwal

Department of Radiology and Imaging

TU Teaching Hospital,

Maharajgunj, kathmandu, Nepal

Email: s1suwal@gmail.com
The most common causes of rim enhancing lesions are metastasis, abscess and high grade glioma. Other causes include tuberculous 
granulomas, neurocysticercosis, sub-acute phase of infarction, demyelinating disease, resolving hematoma and radiation necrosis. ${ }^{1}$ Differentiation of peripheral rim enhancing abscess from the rim enhancing primary tumor or metastasis is difficult using conventional MRI. But the correct and emergent diagnosis is mandatory for the medical or surgical treatment plan of life threatening but treatable conditions like abscesses. ${ }^{2,3,4}$

None of the conventional imaging features are specific in differentiating abscess and tumor necrosis. Advent of Diffusion Weighted Imaging in around 1990s has eased to some extent in differentiating these pathologies but is not totally specific again as there has been reports of atypical DWI of both abscess and tumors. $5,6,7,8,9,10$

So the purpose of this study was to identify features of ring enhancing brain lesions in conventional and DWI MRI, and evaluate the sensitivity and specificity of different MRI parameters in differentiating the rim enhancing intracranial mass lesions using histopathology as gold standard.

\section{METHODS}

After approval from the institutional review board, this retrospective analytical study was conducted in the Department of radiology, The First Hospital of Jilin University, Jilin, China.

All patients who were reported of having rim enhancing brain lesion on MRI from $1^{\text {st }}$
January to $31^{\text {st }}$ December 2012 were searched in electronic medical record. Those patients with Diffusion Weighted Imaging who underwent surgery and pathological diagnosis were coded and included in the study. Fifty one patients with peripheral rim enhancing lesions (58 were lesions) on preoperative MRI were studied. Variables like thin smooth rim enhancement and irregular rim enhancement were studied in contrast enhanced MR. In DWI the signal intensity of the lesion in b0, b1000, ADC map and ADC values from the center were measured.

Pathological results were classified as brain abscess, primary brain tumor, metastasis and neurocysticercosis. Pathological results were further categorized into brain abscess and lesions other than brain abscess. Data was analyzed using SPSS 21.

\section{RESULTS}

Fifty one patients were studied, among them $32(62.7 \%)$ were male and $19(37.3 \%)$ female with age ranging from 4 to 80 and mean age of 48.47 years (Table1).

Out of 58 lesions 50 were supratentorial and 8 were infratentorial, 46 patients had single lesion whereas 5 patients had multiple lesions (Table 2).

Out of 58 lesions studied, $21(36.2 \%)$ were primary tumor, $14(24.1 \%)$ metastasis, $18(31 \%)$ abscesses and 5 (8.6\%) neurocysticercosis (Table 3 ).

Table 1: Demography of rim enhancing lesions

\begin{tabular}{|c|c|c|c|c|}
\hline \multicolumn{2}{|c}{ Age } & Sex & Frequency & Percent \\
\hline Mean & $48.47 \pm 16.83$ & Male & 32 & 62.7 \\
\hline Median Range & 52.00 & Female & 19 & 37.3 \\
\cline { 2 - 5 } & $4-80$ yrs. & Total & 51 & 100 \\
\hline
\end{tabular}

Table 2: Location and Multiplicity of rim enhancing lesions

\begin{tabular}{|c|c|c|c|c|}
\hline Location & Frequency & Percent & Rim enhancing lesions & Frequency \\
\hline Supratentorial & 50 & 86.2 & Single & 46 \\
\hline Infratentorial & 8 & 13.8 & Multiple & 5 \\
\hline
\end{tabular}


Table 3: Pathology of rim enhancing lesions and its frequency

\begin{tabular}{|c|c|c|c|c|}
\hline \multicolumn{2}{|c|}{ Diseases } & \multicolumn{2}{|c|}{ Frequency } & Percent \\
\hline \multicolumn{2}{|c|}{ Abscess } & \multicolumn{2}{|c|}{18} & 31 \\
\hline \multirow{4}{*}{ 蛋 } & Glioblastoma & 15 & \multirow{4}{*}{21} & \multirow{4}{*}{36.2} \\
\hline & Anaplastic oligoastrocytoma & 3 & & \\
\hline & Cellular Schwannoma & 2 & & \\
\hline & Atypical Neurocytoma & 1 & & \\
\hline \multirow{5}{*}{ 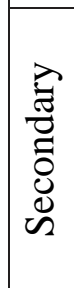 } & Adenocarcinoma Lung & 7 & \multirow{5}{*}{14} & \multirow{5}{*}{24.1} \\
\hline & Small cell carcinoma Lung & 3 & & \\
\hline & Squamous cell carcinoma Lung & 1 & & \\
\hline & Renal Cell carcinoma & 1 & & \\
\hline & Adenocarcinoma Rectum & 1 & & \\
\hline & Neurocysticercosis & 5 & & 8.6 \\
\hline & Total & 58 & & 100 \\
\hline
\end{tabular}

Out of 22 lesions showing uniformly thickened rim, 15 were abscesses, 5 neurocysticercosis, 1 primary brain tumor and 1 metastasis. Whereas, out of 36 irregular rim enhancing lesions, 20 were primary brain tumor, 13 secondaries and 3 abscesses. Using Pearson's chi-square test, significance of this test in differentiating brain abscess and rim enhancing lesions other than brain abscess was $<0.0001$. Sensitivity, specificity, positive predictive value and negative predictive value of this test in differentiating brain abscess from rim enhancing lesions other than brain abscess were respectively $83.33 \%, 82.50 \%$, $68.18 \%$ and $91.67 \%$.

Out of 20 lesions showing restricted diffusion in core of the lesion 16 were abscess and 4 were secondary metastases. Of 38 lesions not showing restricted diffusion 21 were primary brain tumor, 10 metastasis, 5 neurocysticercosis and 2 brain abscesses. Significance of DWI in differentiating abscess from rim enhancing lesions other than brain abscess was $<0.0001$. The sensitivity, specificity, positive predictive and negative predictive value of DWI at center of the lesion in differentiating brain abscess from rim enhancing lesions other than brain abscess were respectively $88.89 \%, 90.00 \%, 80.00 \%$ and $94.74 \%$.

Mean ADC ratio of non-enhancing center to normal white matter in brain abscess, neurocysticercosis, primary brain tumor, and metastasis, were $0.91,2.89,2.98$,

2.07 respectively. AUC in ROC curve for differentiating abscess and non-abscess group is 0.959 with significance of $<0.0001$. At cut off of 1.09 sensitivity, specificity, PPV and NPV of the test in differentiating abscess from non-abscess rim enhancing lesion are respectively $88.9 \%$ and $90 \%$.

Out of 58 rim enhancing lesions, 13 lesions showed restricted diffusion in the center of smooth thin rim enhancing lesion and all were abscesses in pathological examination, whereas in other 35 lesions, 21 were primary brain tumor, 14 metastasis, 5 neurocysticercosis and 5 abscess. Sensitivity, specificity, positive predictive value and negative predictive value of restricted diffusion at non-enhancing core with thin smooth rim in differentiating abscess from non-abscess were respectively $72.22 \%$, $100 \%, 100 \%$, and $88.89 \%$ (Figures 1, 2, 3, 4). 


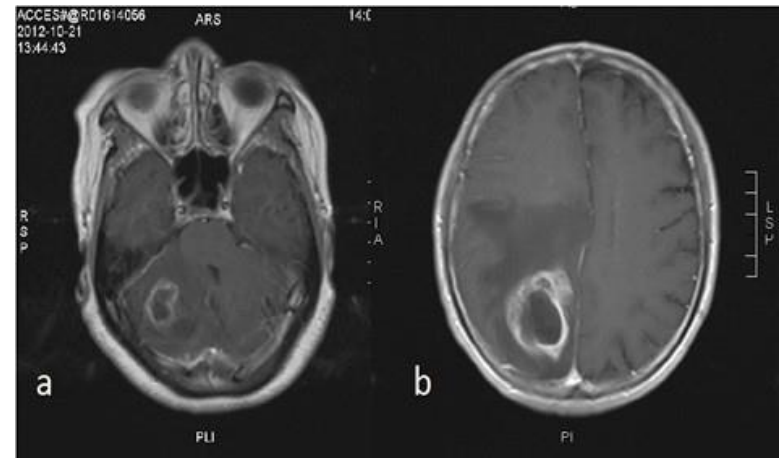

Figure 1: Abscess: Gadolinium enhanced T1WI showing irregular rim at 1a. Right cerebellar hemisphere and $\mathbf{1 b}$. Right cerebral hemisphere.

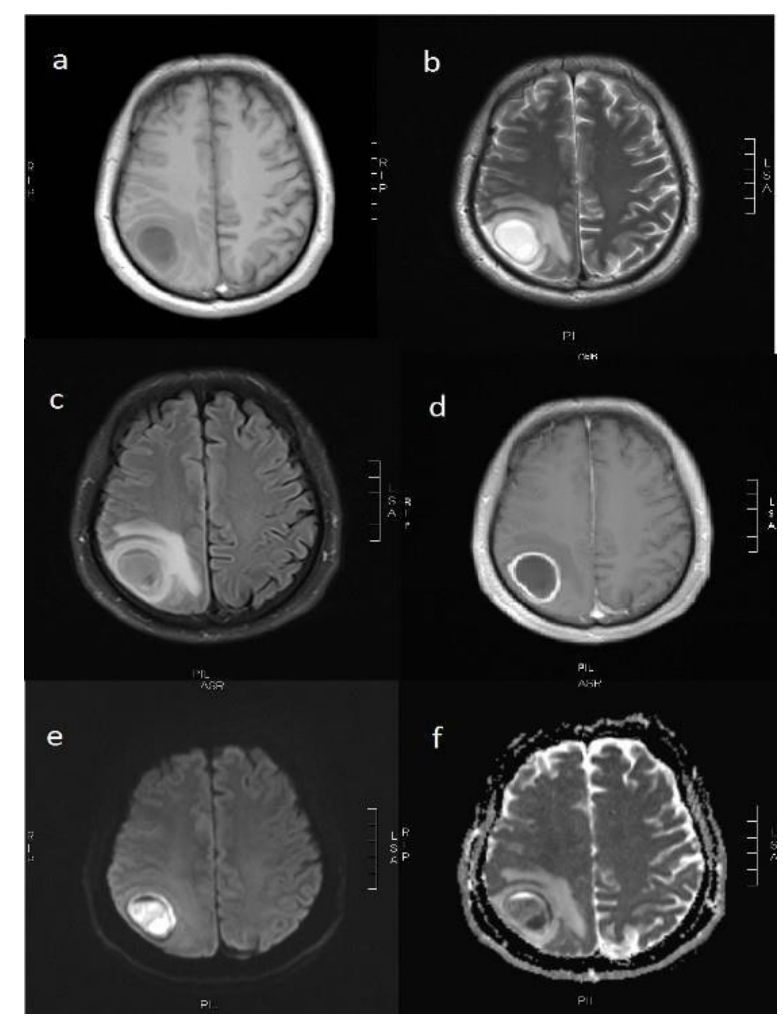

Figure 2: Abscess showing 2a. Hypointense core in T1WI with peripheral white mater hypointensity corresponding to edema $\mathbf{2 b}$. Hyperintense core with T2 hypointense rim with peripheral hyperintense edema $2 c$. FLAIR showing inversion of T2 hyperintense signal of core but not that of edema. $2 d$. GdDTPA enhance TIWI showing smooth thin rim. 2e. DWI showing hyperintense signal corresponding to hypointense ADC. $2 f$. ADC hypointense core.

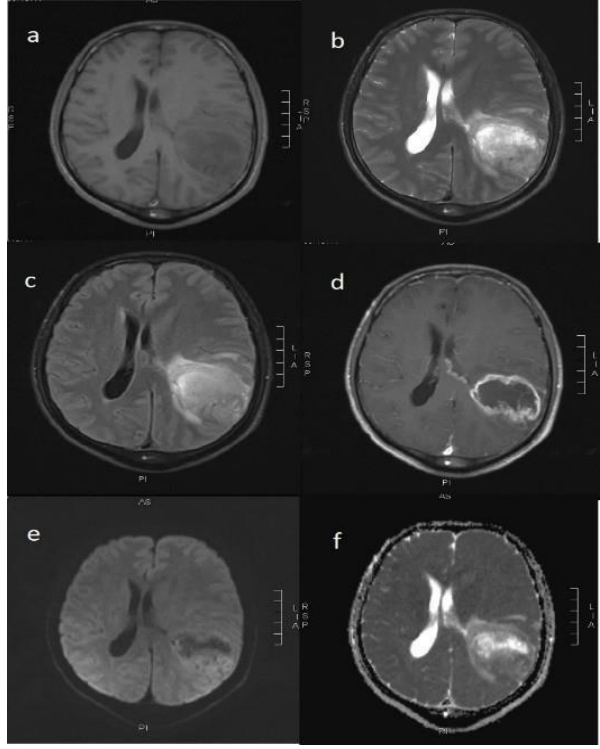

Figure 3: Glioblastoma with thick irregular rim: 3a. T1WI. 3b. T2WI. 3c. FLAIR. 3d. GdDTPA T1WI. 3e. DWI. 3f. ADC Map.

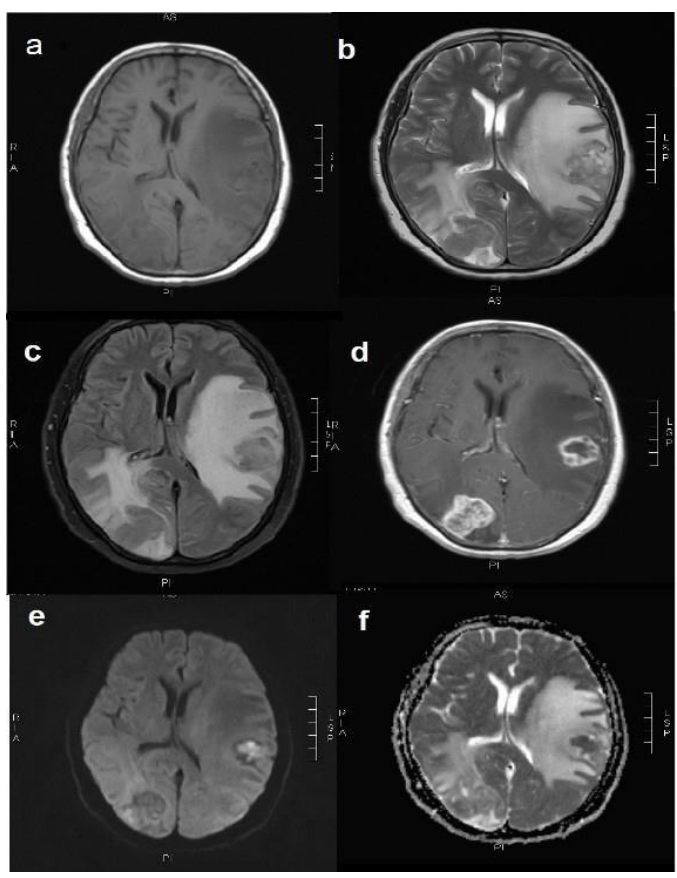

Figure 4: Metastatic adenocarcinoma $4 a$. T1WI showing bilateral lesion, on left frontal lobe with heterogenous mass surrounded by hypointense edema. $\mathbf{4 b}$. Heterogenous mass with surrounding hyperintense vasogenic edema. 4c. FLAIR showing mass isointense to grey mater. $4 d$. Contrast enhanced T1WI showing peripheral rim enhancing lesion with irregularly thickened rim. 4e. DWI showing restricted diffusion at center. $4 f$. ADC map. 


\section{DISCUSSION}

In our study 16 out of 18 abscesses showed complete diffusion restriction in the center with sensitivity of $88.89 \%$ and specificity of $90.24 \%$ in differentiating it from nonabscesses. Two abscesses not showing complete diffusion restriction may be due to late cerebritis stage of the abscess with not well established diffusion restriction in the center or as a result of antimicrobial therapy or due to fungal abscess. ${ }^{11,12,13,14}$ Thirteen out of 16 abscesses showing central diffusion restriction were having thin smooth regular rim enhancement. Three abscesses not showing thin smooth rim can be due to late cerebritis stage of abscess or other causes of abscess like fungal abscess as stated by Luthra $\mathrm{G}$ et al. ${ }^{16}$ Another possibility can be abscess within tumor cavity which already had non-smooth inner surface. ${ }^{11,15,17}$ None of the high grade necrotic tumor and neurocysticercosis in our study showed both diffusion restriction in the center and smooth thin rim. MRS has been helpful in differentiating abscess from high grade necrotic tumors but time consuming and should be reserved for the atypical cases. . $1,19,20$ In this study most of the tumors (all of the glioblastoma and 10 out of 14 metastasis) did not show diffusion restriction. Diffusion restriction in 4 of the metastasis could be due to their internal tissue characteristics. Mucinous secretion of adenocarcinoma of lung, abscess with in the necrotic cyst, fresh hemorrhage can produce restricted diffusion. There was significant correlation of the thick irregular enhancing rim with tumors as compared to abscess in this study. However, diffusion restriction at center was more sensitive and specific as compared to thick irregular enhancing rim to differentiate abscesses from the tumors.

There was a statistically significant difference in the mean non enhancing center to white mater ADC ratio in differentiating abscess from other rim enhancing lesions as seen in majority of the earlier studies with slight difference in the cut-off point and sensitivity and specificity. $3,13,21,22$

In our study of MRI characterization of rim enhancing lesions, we came across certain limitations which were inevitable. Firstly the total number of cases were less and some groups like NCC were even less. In pathological reports no description was found about the content of cyst in case of tumor. By studying the rim enhancing lesions we missed diseases in different stages, specially abscesses and NCC. MRI scans were done in different machines, so to reduce the technical errors we used ratio rather than absolute numbers. Abscess with hemorrhage and tumor with hemorrhage were still difficult to evaluate as the rim characters were not obvious and DWI images were under susceptibility effect of paramagnetic compounds.

\section{CONCLUSIONS:}

On studying different MRI characteristics of rim enhancing lesions, combined approach of rim characteristic with DWI was more helpful in coming to the proper diagnosis rather than relying just on DWI. In atypical cases such as restricted diffusion in center with non-smooth rim, further examinations such as MRS can be helpful in coming to proper diagnosis.

\section{CONFLICT OF INTEREST}

None

\section{SOURCES OF FUNDING}

None

\section{REFERENCES}

1. Brant WE CA. Central Nervous System Neoplasms and tumor like mass. In Brant WE CA. Fundamentals of Diagnostic Radiology; 2007. p. 129-130.

2. Osborne AG. Pyogenic Parenchymal Infections.InDiagnosticNeuroradiology.

3. Alam MS, Sajjad Z, Azeemuddin M, Khan ZA, Mubarak F, Akhtar W. 
Diffusion weighted MR imaging of ring enhancing brain lesion. $J$ Coll Physicians Surg Pak 2012;22(7):428431. Available from: https://ecommons. aku.edu/pakistan_fhs_mc_radiol/3/ [Accessed 15th November 2018].

4. ReicheW,SchuchardtV,HagenT,Il'yasov KA, Billmann P, Weber J. Differential diagnosis of intracranial ring enhancing cystic mass lesions-role of diffusionweighted imaging (DWI) and diffusiontensor imaging (DTI). Clin Neurol Neurosurg 2010;112(3):218-225. https:// doi.org/10.1016/j.clineuro.2009.11.016

5. Duygulu G, Ovali GY, Calli C et al. Intracerebral metastasis showing restricted diffusion: correlation with histopathologic findings. EJR 2010;74(1):117-120. https://doi. org/10.1016/j.ejrad.2009.03.004

6. Holtås S, Geijer B, Strömblad LG, Maly-Sundgren P, Burtscher IM. A ringenhancing metastasis with central high signal on diffusion-weighted imaging and low apparent diffusion coefficients. Neuroradiology 2000;42(11):824-827. https://doi.org/10.1007/s002340000431

7. Mortimer A, O'leary S, Bradley M, Renowden SA. Pitfalls in the discrimination of cerebral abscess from tumour using diffusion-weighted MRI. Clin Radiol 2010;65(6):488-492. https:// doi.org/10.1016/j.crad.2009.12.012

8. Hakyemez B, Erdogan C, Yildirim N, Parlak M. Glioblastoma multiforme with atypical diffusion-weighted MR findings. Br J Radiol 2005;78(935):989-992. https://doi.org/10.1259/bjr/12830378

9. Besada $\mathrm{CH}$, Migliaro $\mathrm{M}$, Christiansen SB, Funes JA, Ajler PM, Mónaco RD. Restricted diffusion in a ringenhancing mucoid metastasis with histological confirmation: case report. JCAT 2010;34(5):770-772. https://doi. org/10.1097/RCT.0b013e3181e7c291

10. Hartmann $M$, Jansen $O$, Heiland S, Sommer C, Münkel K, Sartor
K. Restricted diffusion within ring enhancement is not pathognomonic for brain abscess. AJNR Am J Neuroradiol 2001;22(9):1738-42. Available from: https://ecommons.aku.edu/pakistan fhs_mc radiol/3/ [Accessed 27th June 2018].

11. Olsen WL. Central Nervous System Infections. In Brant WE, Helms CA. Fundamentals of Diagnostic Radiology. 3rd ed.;2007.

12. Cartes-Zumelzu FW, Stavrou I, Castillo M, Eisenhuber E, Knosp E, Thurnher MM. Diffusion-weighted imaging in the assessment of brain abscesses therapy. AJNR Am J Neuroradiol 2004;25(8):1310-1317. Available from:https://ecommons. aku.edu/pakistan_fhs_mc_radiol/3/ [Accessed 13th July 2018].

13. Fanning NF, Laffan EE, Shroff MM. Serial diffusion-weighted MRI correlates with clinical course and treatment response in children with intracranial pus collections. Pediatr Radiol 2006;36(1):26-37. https://doi. org/10.1007/s00247-005-0019-8

14. Mueller-Mang C, Castillo M, Mang TG, Cartes-Zumelzu F, Weber M, Thurnher MM. Fungal versus bacterial brain abscesses: is diffusion-weighted MR imaging a useful tool in the differential diagnosis?. Neuroradiology 2007;49(8):651. https://doi.org/10.1007/ s00234-007-0242-0

15. Mathisen GE, Johnson JP. Brain abscess. Clinical Infectious Diseases 1997;25(4):763-779. $\quad \underline{\text { https://doi. }}$ org/10.1086/515541

16. Luthra $G$, Parihar A, Nath $\mathrm{K}$ et al. Comparative evaluation of fungal, tubercular, and pyogenic brain abscesses with conventional and diffusion MR imaging and proton MR spectroscopy. AJNR Am J Neuroradiol 2007;28(7):1332-1338. $\quad$ https://doi. org/10.3174/ajnr.A0548 
17. Kalita O, Kala M, Svebisova $\mathrm{H}$ et al. Glioblastoma multiforme with an abscess: case report and literature review. J Neurooncol 2008;88(2):221225. https://doi.org/10.1007/s11060008-9557-X

18. Gasparetto EL, Cabral RF, da Cruz LC, Domingues RC. Diffusion imaging in brain infections. Neuroimaging Clin $N$ Am 2011;21(1):89-113. https://doi. org/10.1016/j.nic.2011.01.011

19. Chiang IC, Hsieh TJ, Chiu ML, Liu GC, Kuo YT, Lin WC. Distinction between pyogenic brain abscess and necrotic brain tumour using 3-tesla MR spectroscopy, diffusion and perfusion imaging. BJR 2009;82(982):813-820. https://doi.org/10.1259/bjr/90100265

20. Lai PH, Hsu SS, Lo YK, Ding SW. Role of diffusion-weighted imaging and proton MR spectroscopy in distinguishing between pyogenic brain abscess and necrotic brain tumor. Acta Neurol Taiwan 2004;13:107-13. Available from: https://www.ncbi.nlm. nih.gov/pubmed/15508936\# [Accessed 10th March 2018].

21. Reddy JS, Mishra AM, Behari S et al. The role of diffusion-weighted imaging in the differential diagnosis of intracranial cystic mass lesions: a report of 147 lesions. Surgical neurology 2006;66(3):246-250. $\quad$ https://doi. org/10.1016/j.surneu.2006.03.032

22. Fertikh D, Krejza J, Cunqueiro A, Danish S, Alokaili R, Melhem ER. Discrimination of capsular stage brain abscesses from necrotic or cystic neoplasms using diffusion- weighted magnetic resonance imaging. JNS 2007;106(1):76-81. $\quad$ https://doi. org/10.3171/jns.2007.106.1.76 